\title{
Phase Locking in Millimeter Wave Frequency Synthesizers - Design overview of Charge Pump Phase Locked Loops
}

\author{
NITHIN M \\ SENSE, VIT, Vellore, INDIA \\ Department of ECE \\ HARISH M KITTUR \\ SENSE \\ RV College of Engineering, Bengaluru, INDIA Tamil Nadu, INDIA
}

\begin{abstract}
Phase Locked Loops are key blocks which are widely adopted in all area of electronics, especially transceivers in wireless communication systems. The application of Phase Locked Loop varies from generation of local oscillator signal for upconversion and down conversion, generation and distribution of clock signals and jitter reduction. The most extensive use of Phase Locked Loop is for frequency synthesis. The requirements of synthesizer architectures depend on various system requirements and specifications which are based on regulatory standards. The design of Phase Locked Loop components involves the consideration of various techniques to resolve the nonidealities at front end high frequency components as well as back end low frequency components. This paper presents the background and importance of a Phase Locked Loop, various approaches over the years, design choices for each block and practical design methodology for Charge Pump Phase Locked Loops. This paper also presents the system level design of Phase Locked Loop and supply noise interactions among sub modules inside a charge pump Phase Locked Loop.
\end{abstract}

Key-Words: Phase locking, Charge Pump, Voltage Controlled Oscillator, Frequency dividers, Loop filter Received: June 18, 2019. Revised: May 11, 2020. Accepted: May 29, 2020. Published: June 1, 2020

\section{Introduction}

Phase locking is an important technique widely employed in majority of analog, digital and RF systems. It is an integral part especially in high speed serial links for clock and data recovery as well as in clock synthesizers. Modern processors and system-on-chips utilize multiple Phase Locked Loops (PLL) to cater their varying demands. Conventional PLLs are implemented using Charge Pump architecture as shown in figure 1 .

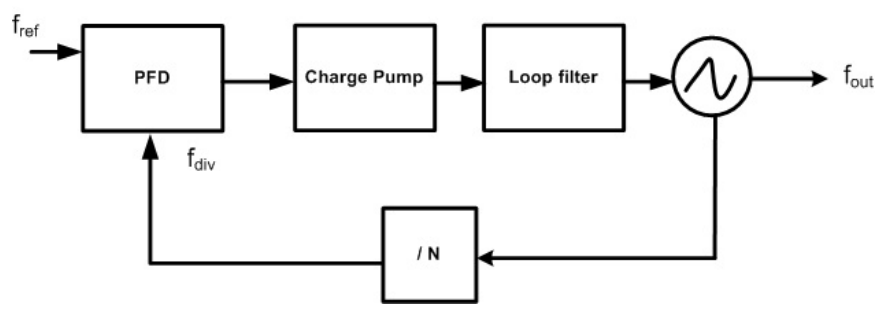

Figure 1: Conceptual diagram of Charge pump PLL

The popularity of charge pump based PLL is because of numerous reasons. Basically it provides flexibility by decoupling various tightly cou- pled parameters like loop bandwidth and damping factor. A plenty of design examples are available in this arena. The most popular references are provided by [1] and [2]. A typical Charge Pump implementation of PLL consists of a phase and frequency detector (PFD), a charge pump (CP), a loop filter (LF), a voltage controlled oscillator (VCO) and divider in feedback to use it for varying applications.

This paper presents an overview of operating principles, basic architecture, and selection of circuit blocks and design methods of Charge Pump Phase Locked Loop based on CMOS technology. The paper is organized as follows. Section 2 gives the fundamentals of Phase Locking, s domain representation of charge pump PLL and frequency synthesizer and figures of merit of PLL. Section 3 and 4 describes the front end and back end components of PLL and its design choices. Section 5 gives the practical design methodology and Section 6 presents system level design of PLL. Finally Section 7 and 8 sums up the state-of-the-art performance of PLLs and supply noise interaction in a charge pump PLL and section 9 concludes the paper. 


\section{Fundamentals of Phase Lock- ing}

PLL is generally characterized in terms of phase and frequency variables. The best analogy to understand PLL is by using a voltage follower as given in figure 2. As the feedback forces Vout to be equal to Vin in a voltage follower, in a PLL feedback forces output and input phases to be equal. Using the phase variables, all the analysis of any feedback systems like stability analysis or noise analysis could be extended to PLLs also [3]-[5].

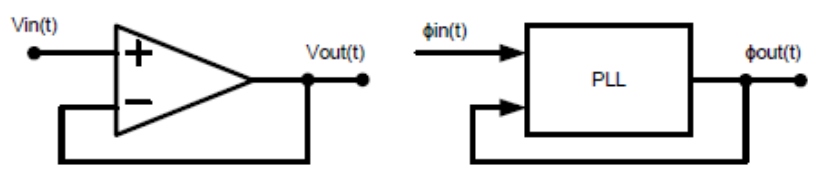

Figure 2: Voltage buffer and PLL

\subsection{Classification of Phase Locked Loops}

PLL architectures are classified basically depending on the crucial block - Voltage Controlled Oscillator (VCO). Depending on the architecture selected for the implementation of a VCO, PLLs are broadly categorized as Wide band PLL and Narrow band PLL[6]-[8].

\subsubsection{Wideband PLL}

Wide-band PLLs use VCOs which are generally classified as relaxation oscillators which are implemented as ring oscillators. The frequency of the oscillator depends on the time constant of the individual inverters, the building block of ring VCOs. Ring VCOs generally have a large tuning range and occupy relatively small on-chip footprint, and potentially low power. However, these benefits are offset by the inferior long-term jitter characteristics and for that reason wide-band PLLs are seldom used in applications with stringent long-term jitter requirements[9]-[10].

\subsubsection{Narrowband PLL}

In Narrow-band PLLs,[11]-[16] VCOs are implemented using resonant characteristics of LC tank circuit. The long-term jitter performance of narrowband PLLs makes them a superior choice over wideband PLLs. At the same time, these types suffer from a very low tuning range which is only $10-20 \%$ of the center frequency. These PLLs consume more area for realizing on chip inductors.

\subsection{PLL as a frequency synthesizer}

PLL fundamentally corrects the phase difference between the two periodic input signals and the output of the PLL is always a multiplication of the input reference frequency[17]. The output frequency or synthesized frequency can be expressed as

$$
f_{\text {out }}=N f_{\text {ref }}
$$

Depending on the $\mathrm{N}$ value, synthesizers are classified as an integer synthesizer or a fractional synthesizer[18]-[22].

\subsection{S domain model of PLL as a fre- quency synthesizer}

Even though PLLs are nonlinear feedback systems, their operation can be approximated by linear analysis using Laplace transforms. All the individual blocks are represented in s domain to get the complete model. The Fig 3 represents the simplified s domain model of synthesizer[23]-[26].

The type and order of PLL is mostly determined by the number of poles located at origin and the number of poles in the system. All VCOs have a pole at origin which makes all PLL atleast type 1 . In order to track the phase, another pole is added by a capacitor $C_{1}$ at dc. This makes the overall PLL a type 2. But the presence of two poles at origin causes instability. So a resistor $R_{1}$ in series with $C_{1}$ capacitor is added which introduces a zero and hence stabilizes the loop. The current pulses from charge pump may further produce ripples in the control line of $\mathrm{VCO}$ [27]-[31]. The ripples deteriorate the spectral purity and generate spurs. This can be minimized by the addition of another capacitor $C_{2}$, which makes the PLL a type 2, third order synthesizer. Higher order and types of PLL are possible, but seldom used because of loop stability issues. The figure 3 shows the s domain representation of a Type II order 3 system.

The phase frequency detector and charge pump are combined and represented by $K_{P F D}$. The loop filter used here is of the order two. $K_{V C O}$, VCO conversion gain represents the tuning or sensitivity of the frequency which is measured in $\mathrm{rad} / \mathrm{s}[32],[33]$. The open loop transfer function 


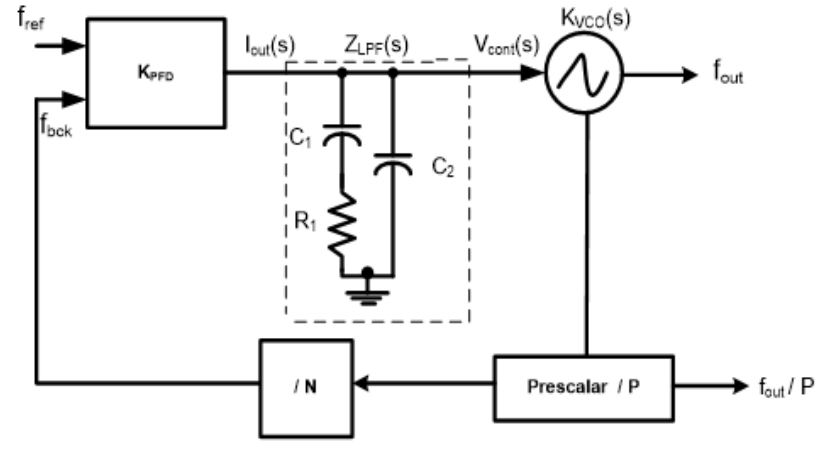

Figure 3: s domain model of PLL as frequency synthesizer

of the synthesizer can be defined as

$$
H_{O L}(s)=\frac{K_{P F D} K_{V C O} Z_{L P F}(s)}{N P s}
$$

\subsection{Figures of merit of a PLL fre- quency synthesizer}

The design of a PLL is a detailed process which involves multitude of variables. In the preliminary design phase, limiting the number of such variables help in deciding the architecture of each block and adopting the technology node. The three fundamental figures of merit considered in the design of PLL are phase noise, spurs, lock time[34]-[39].

\subsubsection{Phase Noise}

It is the random deviation of a frequency tone that is spread around the center frequency. Ideally the tone of a PLL is characterized by an impulse, whereas the real one is spread in the frequency domain shown in the figure 4.A transfer function based study will help to estimate the contribution of phase noise of each block to the total phase noise of the PLL. The figure 5 shows the typical phase noise of PLL components.
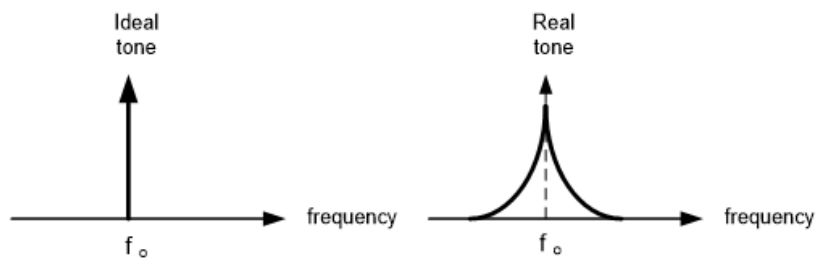

Figure 4: Phase noise effect of a frequency tone

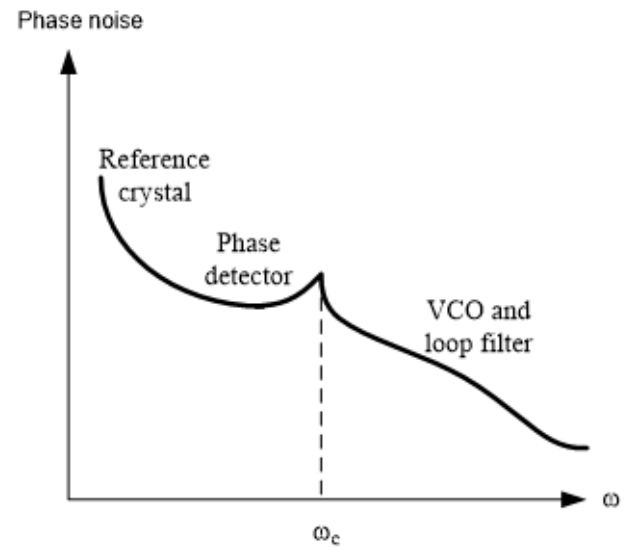

Figure 5: Typical phase noise of PLL components

\subsubsection{Spurious Emissions / Spurs}

The two fundamental reasons for reference spurs are mismatches of currents in a charge pump and the leakage currents. Banerjee [17] and Maxim [18] proposed two models to predict the spurious emissions. The expression proposed by Banerjee is used to calculate the spurs.

$$
\text { Spur }_{l e a k}=\text { Spur }_{b l}+20 \log \frac{\text { Leak }}{K_{\phi}}+\text { Spurgain }
$$

where

$$
\text { Spurgain }=20 \log \frac{K_{v c o} K_{\phi} Z(s)}{s}
$$

\subsubsection{Lock time}

Lock time specifies the maximum time that can be used in the commutation of a channel to another. This is defined by IEEE 802.11a standard and must be less than $1 \mathrm{~ms}$. In a frequency synthesizer lock time is determined by a set of parameters identified in the loop.

\section{PLL front end components and design choices}

The components of a frequency synthesizer operate at varying range of frequencies. The prescalars, which are the first stage frequency dividers and voltage controlled oscillators typically operates at high frequency and are together known as front end high frequency components[40]-[43]. 


\subsection{Prescalars / Frequency dividers}

The first frequency divider (FD) stages in a synthesizer are called as prescalars. They are not treated separately for a low frequency synthesizer, but for a mm wave synthesizer, prescalar needs to operate at the highest frequency of the loop and thus its design is challenging.

The figure 6 shows the basic classification of prescalar for $\mathrm{mm}$ wave frequencies. It is categorized as analog, digital and a combination of both known as hybrid. The choice of different topologies depends on various requirements like power consumption, locking range, phase noise and complexity of design.

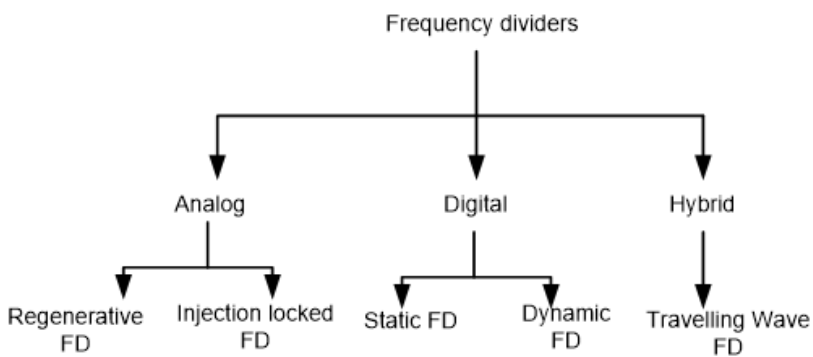

Figure 6: Design choices of prescalars

\subsubsection{Analog frequency dividers}

The basic analog frequency divider if first proposed in [44] is a regenerative type where it employs a mixer based on a Gilbert cell. These types of dividers does not self oscillate. Moreover the performances of these dividers are not satisfactory above $60 \mathrm{GHz}$ [45-50]. The locking range which is based on eqn 1 is less for these types and power requirement is also more.

$$
\text { Lockingrange }(\%)=\frac{f_{\max }-f_{\min }}{f_{\text {center }}} \times 100
$$

The promising analog divider which is widely used is of injection locking type where the oscillators are forced to oscillate from its resonant frequency. By properly choosing the amplitude and frequency of the sinusoid, the circuit will oscillate at the injection frequency; the figure 7 shows the injection locking principle. These dividers can be adopted for high frequency division but at the cost of lock range and design complexity.

\subsubsection{Digital frequency dividers}

The digital dividers are of two types - static and dynamic. Static dividers are mostly based on edge

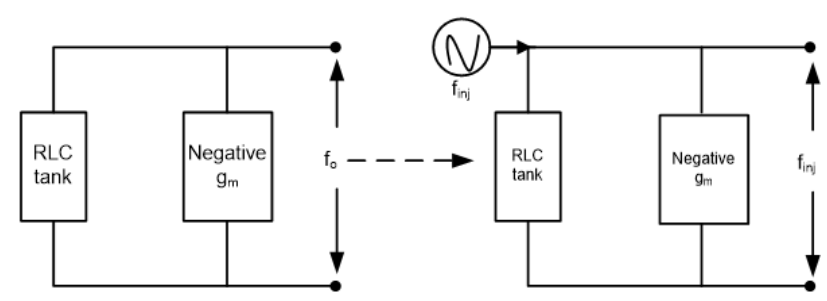

Figure 7: Principle of injection locking

triggered flip flops in a negative feedback loop. The maximum operating frequency of a static FD is given by

$$
f_{\max }=\frac{1}{2 \tau_{p d}}
$$

where $\tau_{p d}$ is the propagation delay from input to output. [25]- [27] Dynamic dividers are different from static in the use of latches and true single phase clock dividers in the circuit topology. If static is good for better locking range, dynamic is good for its low power consumption.

\subsubsection{Travelling Wave frequency dividers}

This topology was first proposed in [51] with bipolar technologies and later with CMOS technologies. This topology offers many advantages like differential outputs and less number of transistors leading to less parasitics.

The figure 8 shows a travelling wave FD, where it consists of three differential amplifiers. The operation resembles that of a bistable differential amplifier that can switch between master and slave state. The analog nature of the circuit can be identified by studying the current transitions between different branches of the circuit.

\subsection{Voltage Controlled Oscillator}

Voltage Controlled Oscillator (VCO) [52]-[53],[63] is considered as the heart of any synthesizer that operates at the highest frequency. It provides the actual oscillations at the output. The spectral purity of the synthesizer depends on VCO and its ability to reject common mode noise. The lock range of the PLL is determined by the tuning range of VCO. Figure 9 shows the classification of VCOs [54].

Ring oscillator is a popular resonator less VCO, where it uses cascade stages of inverters. The tuning of these kinds of oscillators is done by varying the transconductance of the delay stages. 


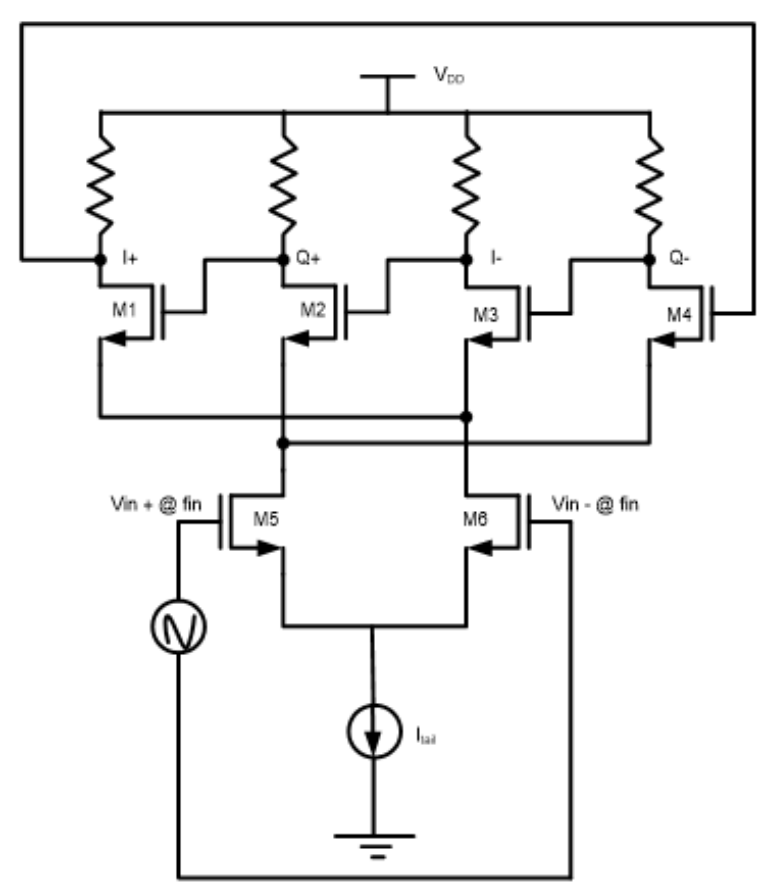

Figure 8: Travelling wave frequency divider

Typically a tuning range of more than $30 \%$ is possible with these types. But tuning can be achieved at the cost of spectral purity and phase noise performance. The ring oscillators reported are limited to a maximum of $15 \mathrm{GHz}$ in bulk CMOS processes and therefore not a choice for $\mathrm{mm}$ wave frequency synthesizers [55].

Another category is resonator based VCO [54], where a tank circuit is employed. The main advantages of using a resonator based VCO is it can work close to the maximum frequency $f_{\max }$ of a given technology [56]. The frequency of oscillation is determined by

$$
f_{\text {osc }} \approx \frac{1}{2 \sqrt{L C}}
$$

where $\mathrm{L}$ and $\mathrm{C}$ are the inductance and capacitance. The design of oscillator with low phase noise can be done using the Leeson's phase noise model as shown in figure 10 [57] - [59]. This model shows the variation of phase noise based on the frequency offset, center frequency and the $\mathrm{Q}$ factor of the LC tank.

$L(\Delta \omega)=10 \log \left[\frac{2 k T}{P_{\text {sig }}} F\left(1+\left(\frac{\omega_{o}}{2 Q \Delta \omega}\right)^{2}\right)\left(1+\frac{\Delta \omega}{\Delta \omega}\right]\right.$

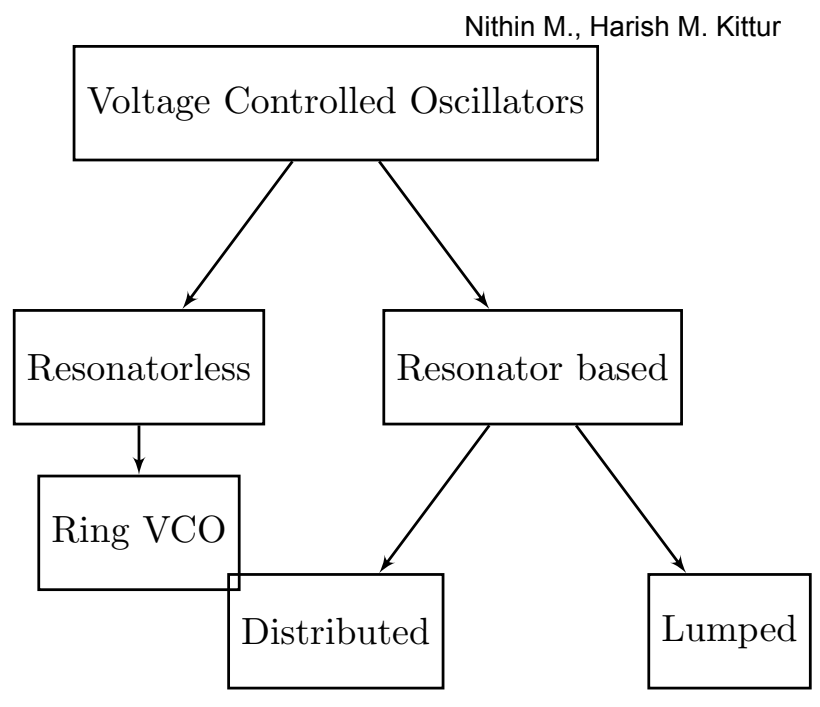

Figure 9: Design choices for VCO

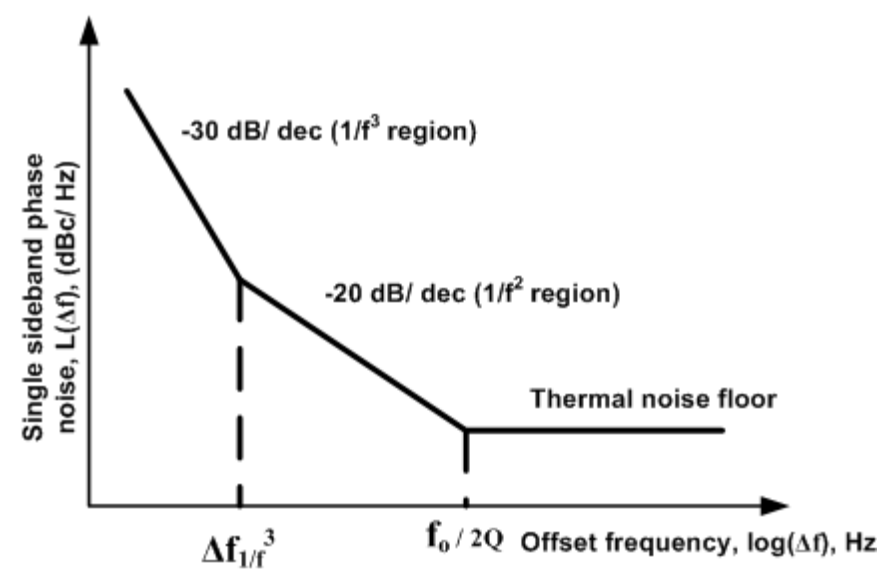

Figure 10: Leeson's phase noise model

The circuit in figure 11 shows a CMOS based $\mathrm{VCO}$. The oscillation frequency is varied by the tuning mechanism and is usually accomplished by the capacitors.

$$
\text { Tuningrange }=\frac{\Delta f}{f_{\text {center }}} \times 100
$$

The voltage swing in the CMOS cross-coupled oscillator is twice the voltage swing in the NMOS cross-coupled oscillator. From (1), the phase noise decreases as the voltage swing increases. In order to have lower phase noise, CMOS topology is better than NMOS type. 


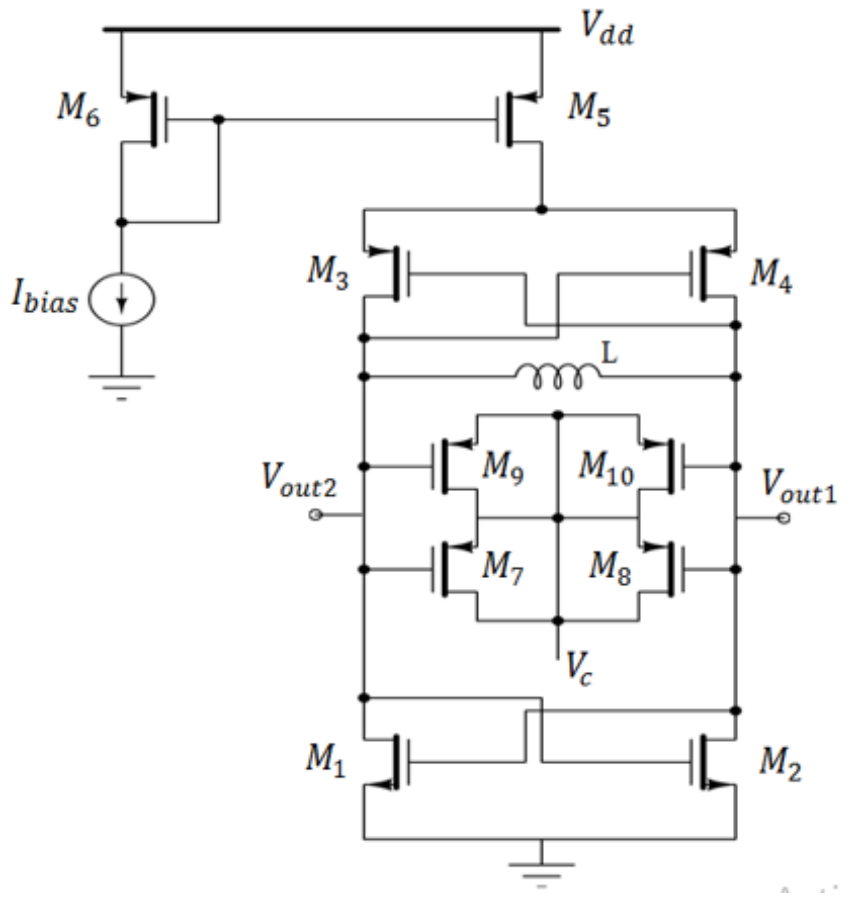

Figure 11: CMOS Cross coupled oscillator

\section{PLL Back end Components \& design choices}

Compared to front end components, PLL back end components including Phase Frequency Detector, Charge pump and feedback divider chain operates at lower frequencies. This section presents an overview and design choices for back end circuits.

\subsection{Feedback divider}

Feedback divider generates an output which is very close to synthesizer reference frequency. The input for the divider chain comes from the prescalar. In most of the cases the prescalar frequencies will be in the range of $\mathrm{GHz}$ and feedback dividers need to divide those frequencies into $\mathrm{MHz}$ range to get close to reference frequency.

A dual modulus divider is a widely used circuit to change the division ratio in a frequency synthesizer. These circuits are used when area is a concern and are commonly implemented in MOS Current Mode Logic MCML or Source Coupled Logic (SCL). The different approaches in the design of CML dividers relate the gate delay to the power consumption, with the constraint of a given output swing and voltage gain of the differential pairs, in order to satisfy the requirement on the noise margin. Phase noise is an impor- tant criterion in the design of dividers, as there is a tight compromise between low phase noise and high frequency of operation. A popular CML based topology is shown in figure 12 .

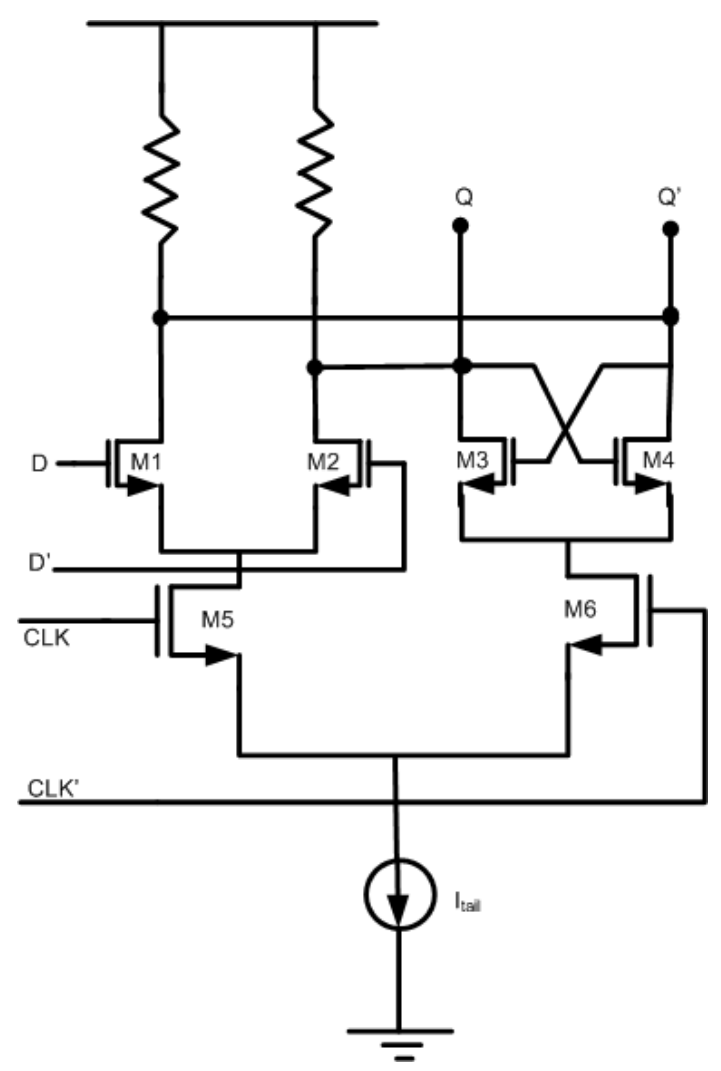

Figure 12: CML based divider

\subsection{Phase Frequency Detector, Charge Pump and Loop filter}

The components PFD, CP and loop filter forms the rest of the PLL back end whose combined task is to provide a stable dc tuning voltage to the VCO so that synthesizer can move towards lock state.

PFD is a circuit which detects both the phase and frequency between two signals and generates an output in proportion to them. The simple and widely used implementation is with two edge triggered D flip flops.

\subsubsection{Practical considerations for the de- sign of PFD}

Three fundamental steps are followed in the design of a PFD.

1. The delay estimation of the reset latch

2. Proper scaling of the logic gates 
3. Selection of the delay block

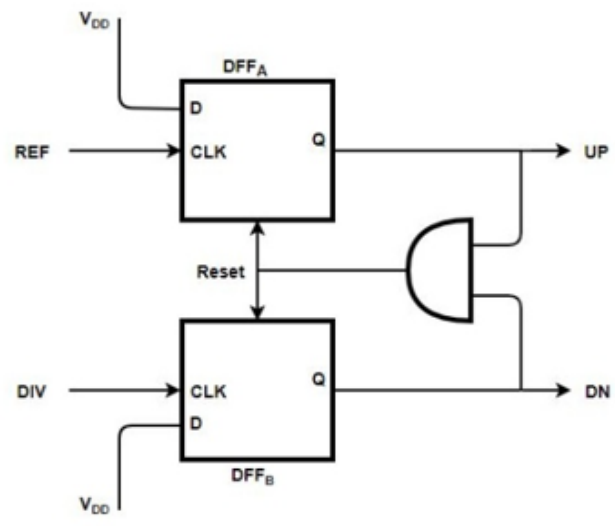

Figure 13: Phase Frequency Detector

PFD implementation has a potential problem known as dead zone problem when the phase error is very small which leads to incorrect operation of subsequent stages or even zero loop gain. This dead zone prevents the synthesizer to work until the phase error reaches a certain minimum value. Many PFD implementations are reported [59-61] aimed at robust designs in improving the various characteristics of PFD like operating frequency, dead zone, complexity, and symmetry. The next component in the line is the charge pump which converts the output signal of PFD into a charge and thereby responsible for moving control voltage of VCO up or down by pumping current in or out of the loop filter as shown in figure 14 .

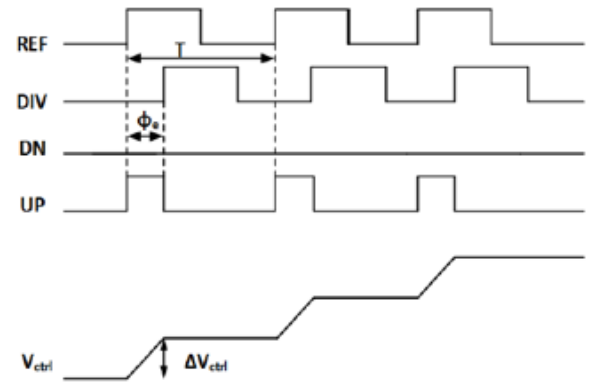

Figure 14: Charge pump output

The increase in $V_{c t r l}$ is given by

$$
\Delta V_{c t r l}=\frac{I_{c p}}{C_{p}} \frac{\Phi_{e}}{2 \pi} T
$$

The average charge dumped per cycle is given by

$$
Q_{c t r l}=C_{p} \frac{\Delta V_{c t r l}}{T}=\frac{I_{c p}}{2 \pi} \Phi_{e}
$$

The transfer function of the PFD and the charge pump is given by

$$
P D(s)=K_{P D}=\frac{I_{C P}}{2 \pi}
$$

\subsubsection{Practical considerations for the de- sign of charge pump}

The design of charge pump is done in such way that reduces mismatches between up and down currents and thereby nullifies charge deposition at the loop filter. Fluctuations or variations in the filter voltage due to current mismatch in the charge pump result in reference spurs or discrete spurs in the output spectrum of the frequency synthesizer, called as reference feedthrough. In addition, to maintain a constant loop-bandwidth, the magnitude of the charge pump output currents must be independent of the output voltage.

Authors in [5] proposed a charge pump for low- voltage PLLs that combines a replica biasing technique and a feedback structure. Current matching is improved over a wide output voltage range. Apart from the mismatches, output impedance of the charge pump is also considered invariably.

The loop filter, the last circuit in the back end, suppresses the high frequency components at the charge pump output. The overall loop dynamics and stability depends on loop filter. . It consists of a resistor in series with a capacitor $C_{1}$ both of which are in parallel with another capacitor $C_{2}$. The capacitor $C_{1}$ introduces a pole which is balanced by the resistor introducing a zero so that it is stable in the given frequency range. The addition of $C_{2}$ is to suppress the ripples in the control voltage, $V_{c t r l}$. This may lead to the system becoming unstable. Hence, the capacitor and resistor values are chosen such that they ensure optimum stability and maximum high frequency noise component rejection. The transfer function of the loop filter is given by

$$
F(s)=\frac{s+\frac{1}{R C_{1}}}{s+\frac{C_{1}+C_{2}}{R C_{1} C_{2}} s C_{2}}
$$

The schematic of PFD-CP-LF is as shown in figure 15 . 


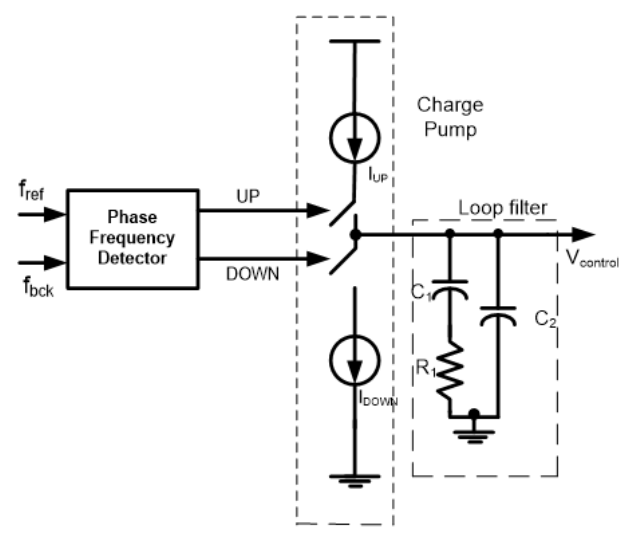

Figure 15: PFD CP Loop Filter

\section{Practical Design Methodol- ogy}

The most crucial part in the design of a frequency synthesizer is the determination of practical and realizable specifications for the back end and front end circuits. This section proposes a practical design methodology to determine the initial requirements for the various specifications that need to be considered for the design. Table 1 shows specifications to be targeted in the design of a $\mathrm{mm}$ wave frequency synthesizer.

Table 1: Target specification of millimeter wave frequency synthesizer

\begin{tabular}{|c|c|l|}
\hline \multirow{2}{*}{ Frequency Synthesizer } & \multicolumn{2}{|c|}{ Locking range } \\
\cline { 2 - 3 } & \multicolumn{2}{|c|}{ Settling time } \\
\cline { 2 - 3 } & \multicolumn{2}{|c|}{ In band phase noise } \\
\cline { 2 - 3 } Front end circuits & Prescalar & Locking range \\
\cline { 2 - 3 } & \multirow{2}{*}{ VCO } & Phase noise \\
\cline { 2 - 3 } & & Tuning range \\
\hline \multirow{2}{*}{ Back end circuits } & Feedback divider & Division ratio \\
\cline { 2 - 3 } & PFD & Dead zone \\
\hline
\end{tabular}

\subsection{Identifying Initial requirements of a synthesizer}

The first step in the design of a synthesizer is to develop the initial requirements. A sample initial requirement for a $\mathrm{mm}$ wave synthesizer is as shown in Table 2.
Table 2: Initial requirements of frequency synthesizer

\begin{tabular}{|c|c|}
\hline Initial requirement & Unit or relevant data \\
\hline Application & WLAN / Wicomm \\
\hline Technology & Standard CMOS 90 nm \\
\hline Output frequency & $\mathrm{GHz}$ \\
\hline Reference frequency & $\mathrm{MHz}$ \\
\hline Reference accuracy & $\mathrm{ppm}$ \\
\hline Phase noise @ 1 MHz & $\mathrm{dBc} / \mathrm{Hz}$ \\
\hline Reference spurious level & $\mathrm{dBc}$ \\
\hline Settling time & $\mathrm{\mu s}$ \\
\hline Supply Voltage & $\mathrm{V}$ \\
\hline Power & $\mathrm{mW}$ \\
\hline
\end{tabular}

\subsection{Architectural selection}

With the initial requirements, architecture selection can be done for both front end and back end circuits. Depending on the target specifications architectures can be selected and simulated in block level and later.

\subsection{Synthesizer integration}

The overall circuits can be simulated in any of the available CAD tools. Transistor level simulation of complete synthesizer is a tedious process. Due to the large simulation time, front end and back end circuits are simulated separately before integration. The figure 16 shows practical methodology for the design of synthesizer. It is a good practice to start with the system level simulation before implementing individual blocks in circuit level.

\section{System level design of PLL}

This section discusses the system level design of PLL using the CPPSim toolkit. The CPPSim package includes two tools namely, PLL Design Assistant and Sue2. Initially the designing is done using PLL Design Assistant which considers the ideal conditions. Further, Sue2 is used to complete the system level design by including the non-idealities like charge pump mismatch and non-zero phase error.

A black box approach in order to understand the operation of PLL is performed using the PLL Design Assistant tool by CPPSim software. The PLL design assistant tool provides a graphical user interface to design PLL at the transfer function level. This section presents the analysis of results obtained using design assistant tool. 


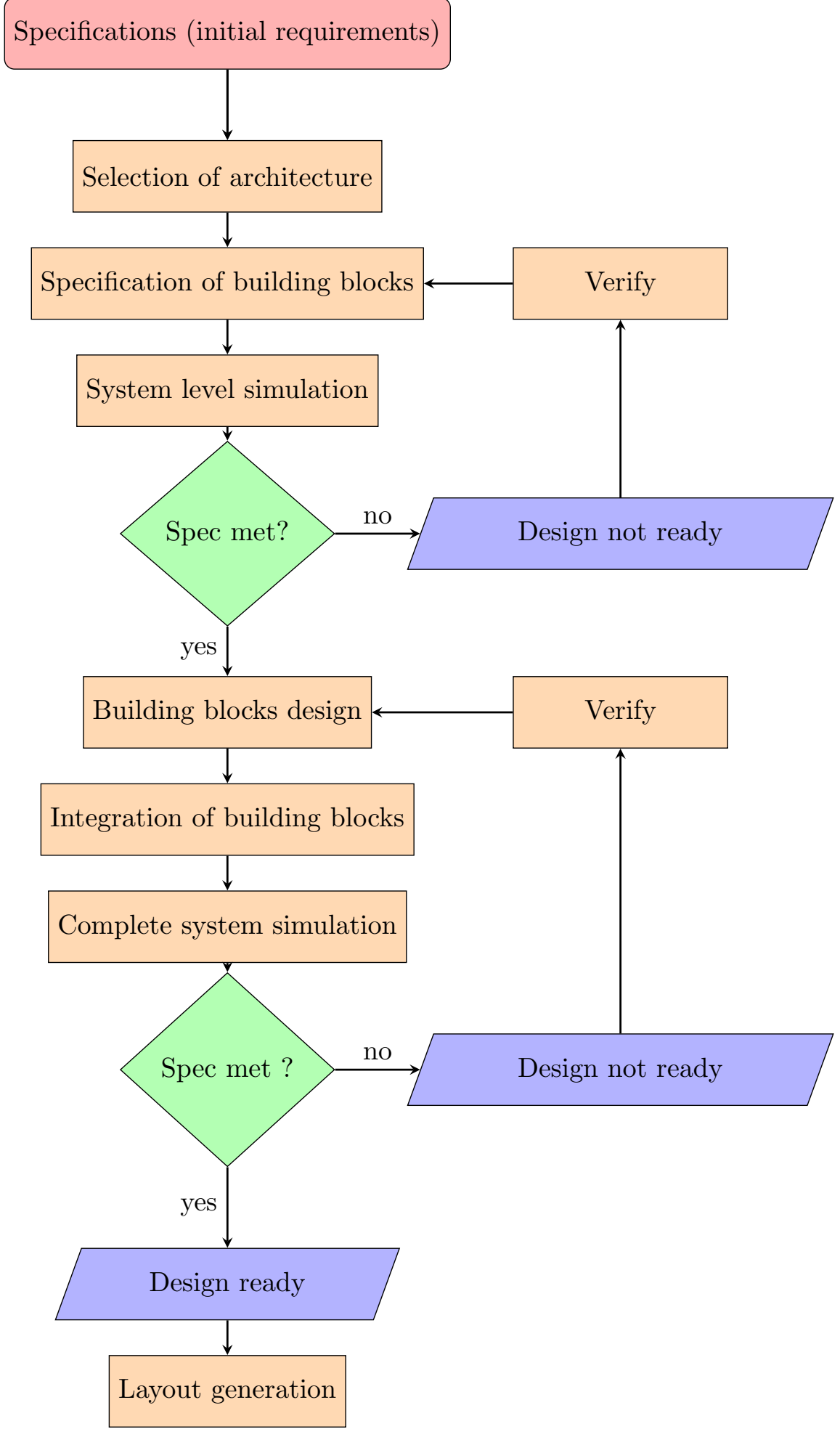

Figure 16: Practical design methodology 


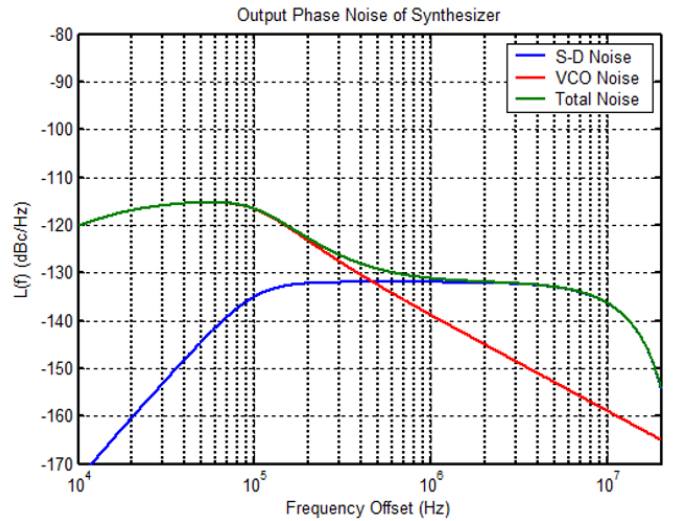

Figure 17: Phase noise plot

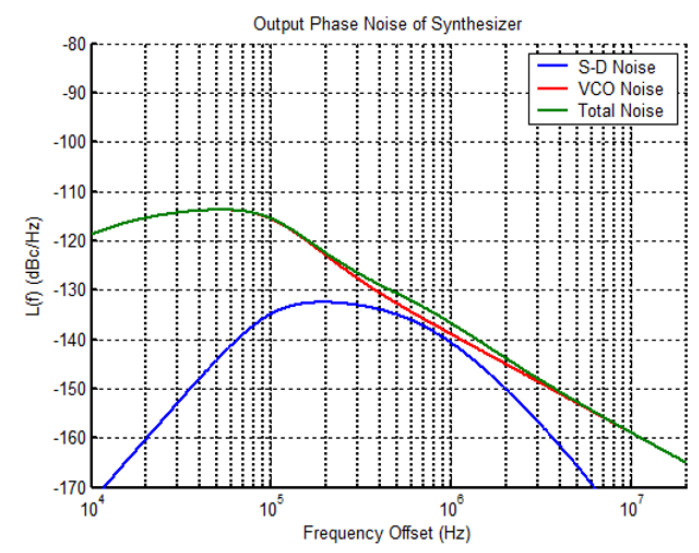

Figure 18: Phase noise plot with addition of parasitic poles

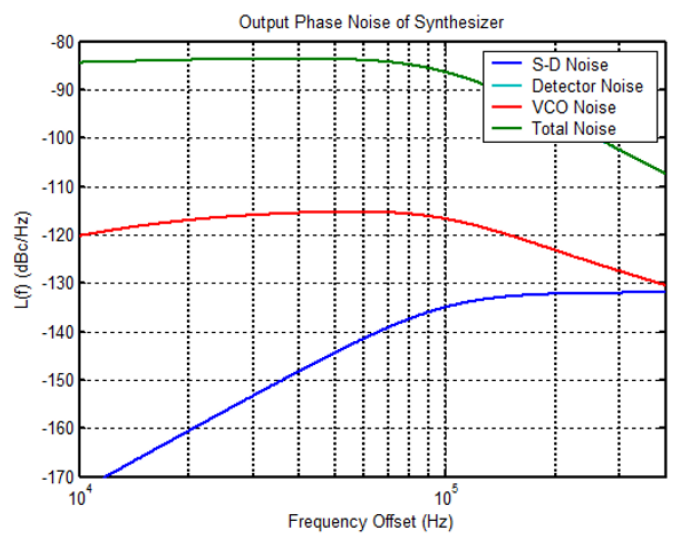

Figure 19: Phase noise plot with $-90 \mathrm{dBc} / \mathrm{Hz} \mathrm{PFD}$ referred noise

With the PLL design assistant, noise performance of the PLL under different configurations was examined and we decided on the system parameters needed to meet the GSM phase noise specifications and also to meet the settling time requirements. Also, the impact of parameter variations was analyzed along with its effects on phase noise and settling time. Fig 17 to Fig 19 shows the results of phase noise under various conditions and closed loop step response and stability analysis of a PLL designed with PLL design assistant tools.

\section{State-of-the-art in CMOS PLL}

This section compares different CMOS frequency synthesizers implemented so far in diverse technology processes. An overview of recent state-ofthe-art synthesizers representing their technology node and fundamentals figures of merit is given in Table 3. The frequency is expressed in Giga Hertz, technology node in $\mathrm{nm}$, phase noise measured at $1 \mathrm{MHz}$ offset in $\mathrm{dBc}$ per $\mathrm{Hz}$ and power in milli watts.

Table 3: State-of-the-art CMOS frequency synthesizer

\begin{tabular}{|c|c|c|c|c|c|}
\hline Ref & $V_{D D}$ & f & Tech & PN & P \\
\hline 10 & 1.2 & 5.5 & $130 \mathrm{~nm}$ & -116.7 & 36 \\
\hline 22 & 1 & 5.5 & $180 \mathrm{~nm}$ & -115.7 & 27.5 \\
\hline 23 & 1.8 & 5.2 & $180 \mathrm{~nm}$ & -116.2 & - \\
\hline 24 & 1.8 & 3.5 & $180 \mathrm{~nm}$ & -120.7 & - \\
\hline 35 & 1.8 & 5.2 & $180 \mathrm{~nm}$ & -114.2 & - \\
\hline 36 & 2.5 & 4.3 & $250 \mathrm{~nm}$ & - & 117.5 \\
\hline 47 & 1.8 & 5.2 & $180 \mathrm{~nm}$ & -119.2 & - \\
\hline 58 & 2.5 & 4 & $250 \mathrm{~nm}$ & -114 & 180 \\
\hline 59 & 1.5 & 4.9 & $250 \mathrm{~nm}$ & -104.7 & 25 \\
\hline
\end{tabular}

\section{Supply noise interactions in charge pump PLL}

The output noise induced by supply interactions are mainly contributed by the exchange of signals between each sub block and power grid. There are typically five types of signal exchange as indicated in the figure as

1. supply current vs input voltage,

2. all supply currents of sub blocks super impose on the power grid

3. output voltage vs input voltage

4. supply current vs output voltage

5. supply current vs noise to the supply voltage 


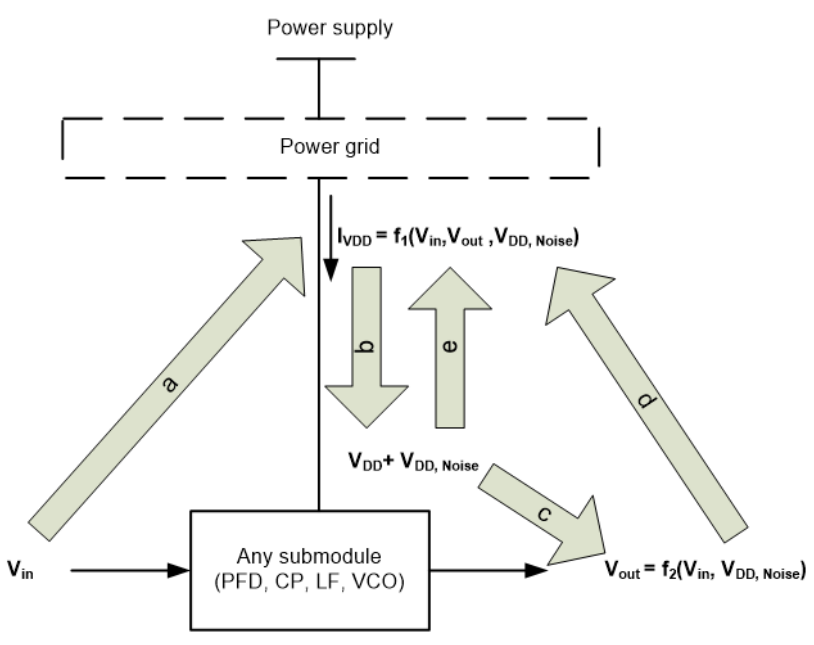

Figure 20: Supply noise interaction in PLL

\section{Conclusion}

Phase locking as described in this paper is one of the inevitable approaches for frequency synthesis. This paper presented the background and importance of a Phase Locked Loop, various approaches over the years, system level design approaches, practical design methodology, architectures for Charge Pump PLLs. The system level design of a Charge Pump Phase locked loop was presented with PLL design assistant tool and supply noise interactions among sub modules inside a charge pump PLL are also discussed.

\section{References}

[1] Valla, M., et al., "A 72-mW CMOS 802.11a Direct Conversion Front-End with 3.5-dB NF and 200-KHz 1/f Noise Corner," IEEE Journal of Solid- State Circuits, Vol. 40, No. 4, April 2005, pp. 70-977.

[2] Zhang, P., et al., "A 5-GHz DirectConversion CMOS Transceiver," IEEE Journal of Solid-State Circuits, Vol. 38, No. 12, December 2003, pp. 2232-2237.

[3] Zargari, M., et al., "A Single-Chip DualBand Tri-Mode CMOS Transceiver for IEEE 802.11a/b/g Wireless LAN," IEEE Journal of Solid-State Circuits, Vol. 39, No. 12, December 2004, pp. 2239-2249.

[4] Zhang, P., et al., "A Single-Chip Dual-Band Direct-Conversion IEEE 802.11a/b/g WLAN
Transceiver in $0.18 \mu \mathrm{m}$ CMOS," IEEE Journal of Solid-State Circuits, Vol. 40, No. 9,September 2005, pp. 1932-1939.

[5] Craninckx, J., and M. Steyaert, Wireless CMOS Frequency Synthesizer Design, Norwell, MA: Kluwer Academic Publishers, 1998.

[6] Zannnoth, M., et al., "A Fully Integrated VCO at $2 \mathrm{GHz}$," IEEE Journal of Solid-State Circuits, Vol. 33, No. 12, December 1998, pp. 1987-1991.

[7] Vassiliou, I., et al., "A Single-Chip Digitally Calibrated $5.15-5.825-\mathrm{GHz} \quad 0.18 \mu \mathrm{m}$ CMOS Transceiver for 802.11a Wireless LAN," IEEE Journal of Solid-State Circuits, Vol. 38 , No. 12, December 2003, pp. 2221-2229.

[8] Ahola, R., et al., "A Single-Chip CMOS Transceiver for 802.11a/b/g Wireless LANs," IEEE Journal of Solid-State Circuits, Vol. 39, No. 12, December 2004, pp. 2250-2258.

[9] Lam, C., and B. Razavi, "A 2.6-GHz/5.2GHz Frequency Synthesizer in $0.4 \mu \mathrm{m}$ CMOS Technology," IEEE Journal of Solid-State Circuits, Vol. 35, No. 5, May 2000, pp. 788-794.

[10] Bhattacharjee, J., et al., "A 5.8 GHz Fully Integrated Low Power Low Phase Noise CMOS LC VCO for WLAN Applications," 2002 IEEE MTT-S Int. Microwave Symp. Dig., Vol. 1, February 2002, pp. 585-588.

[11] Chu, Y., and H. Chuang, "A Fully Integrated

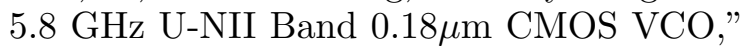
IEEE Microwave and Wireless Components Letters, Vol. 13, No. 7, July 2003, pp. 287-289.

[12] Hajimiri, A., and T. H. Lee, "Design Issues in CMOS Differential LC Oscillators," IEEE Journal of Solid-State Circuits, Vol. 34, No. 5, May 1999, pp. 717-724.

[13] Ham, D., and A. Hajimiri, "Concepts and Methods in Optimization of Integrated LC VCOs," IEEE Journal of Solid-State Circuits, Vol. 36, No. 6, June 2001, pp. 896-908.

[14] Hajimiri, A., and T. H. Lee, The Design of Low Noise Oscillators, Norwell, MA: Kluwer Academic Publishers, 2000. 
[15] Herna'ndez, J., et al., "Analysis of Architectures for $1.8 \mathrm{GHz}$ CMOS LC-Tank Voltage- Controlled Oscillators," Proceedings of DCIS'99, Palma de Mallorca, November 1999, pp. 139-142.

[16] Egazi, E., H. Sjo"land, and A. Abidi, "A Filtering Technique to Lower LC Oscillator Phase Noise," IEEE Journal of Solid-State Circuits, Vol. 36, No. 12, December 2001, pp. 1921-1930.

[17] Kucera, J. J., "Wideband BiCMOS VCO for GSM/UMTS Direct Conversion Receivers," IEEE International Solid-State Circuits Conference, Vol. 44, February 2001, pp. $374-375$.

[18] Y. Sun, L. Siek, P. Song, Design of a high performance charge pump circuit for low voltage phase-locked loops, in: ISIC'07, 2007, pp. 271-274.

[19] B. Razavi, A study of injection locking and pulling in oscillators. IEEE J. Solid-State Circuits 39(9), 1415-1424 (2004)

[20] W. Hui, Z. Lin, A $16-$ to- $18 \mathrm{GHz} 0.18 \mu \mathrm{m}$ Epi-CMOS divide-by-3 injection-locked frequency divider. IEEE International SolidState Circuits Conference, Feb. 2006, pp. 2482-2491

[21] W. Hui, A. Hajimiri, A 19 GHz 0.5 mW 0.35 $\mathrm{mm}$ CMOS frequency divider with shuntpeaking locking-range enhancement. IEEE International Solid-State Circuits Conference, Feb. 2001, pp. 412-413, 471

[22] R. Adler, A study of locking phenomena in oscillators. Proc. IRE 34(6), 351-357 (1946)

[23] S. Verma, H.R. Rategh, T.H. Lee, A unified model for injection-locked frequency dividers. IEEE J. Solid-State Circuits 38(6), 1015-1027 (2003)

[24] H.R. Rategh, T.H. Lee, Superharmonic injection-locked frequency dividers. IEEE J. Solid- State Circuits 34(6), 813-821 (1999)

[25] B. Razavi, Design of Integrated Circuits for Optical communications (McGraw-Hill Science, New York, 2002)

[26] P. Andreani, A. Bonfanti, L. Romano, C. Samori, Analysis and design of a $1.8-\mathrm{GHz}$ CMOS LC quadrature VCO. IEEE J. SolidState Circuits 37(12), 1737-1747 (2002)
[27] X.P. Yu, H.M. Cheema, R. Mahmoudi, A. van Roermund, X.L. Yan, A $3 \mathrm{~mW} 54.6$ $\mathrm{GHz}$ divide-by-3 injection locked frequency divider with resistive harmonic enhancement. IEEE Microw. Wireless Components Lett. 19(9), 575-577 (2009)

[28] J. Lin, M. Jian-Guo, S.Y. Kiat, A.D. Manh, 9.3-10.4-GHz-band cross-coupled complementary oscillator with low phase-noise performance. IEEE Trans. Microw. Theory Tech. 52(4), 1273-1278 (2004)

[29] J. Jin, X.P. Yu, J.J. Zhou, T.T. Yan, Gigahertz range injection locked frequency dividers with band-width enhancement and supply rejection. IET Electron. Lett. 44(17), 999-1000 (2008)

[30] D. Mijuskovic, M. Bayer, T. Chomicz, N. Garg, F. James, P. McEntarfer, J. Porter, Cellbased fully integrated CMOS frequency synthesizers. IEEE J. Solid-State Circuits 29(3), 271-279 (1994)

[31] C. Hsien-Ku, C. Da-Chiang, J. Ying-Zong, L. Shey-Shi, A 30-GHz wideband low power CMOS injection-locked frequency divider for 60-GHz wireless-LAN. IEEE Microw. Wireless Components Lett. 18(2), 145-147 (2008)

[32] H.K. Chen, C. Hsien-Jui, D.C. Chang, Y.Z. Juang, Y. Yu-Che, S.S. Lu, A mm-wave CMOS multimode frequency divider. IEEE International Solid-State Circuits Conference, Feb. 2009, pp. 280-281,281a

[33] D.J. Cassan, J.R. Long, A 1-V transformerfeedback low-noise amplifier for $5-\mathrm{GHz}$ wireless LAN in $0.18 \mathrm{~mm}$ CMOS. IEEE J. SolidState Circuits 38(3), 427-435 (2003)

[34] C. Jun-Chau, L. Liang-Hung, 40GHz widelocking-range regenerative frequency divider and low-phase-noise balanced VCO in $0.18 \mathrm{~mm}$ CMOS. IEEE International SolidState Circuits Conference, Feb. 2007, pp. 544-621

[35] L. Shao-Hua, S.L. Jang, C. Yun-Hsueh, A low voltage divide-by-4 injection locked frequency divider with quadrature outputs. IEEE Microw. Wireless Components Lett. 17(5), 373-375 (2007)

[36] L. Tang-Nian, Y.J.E. Chen, A 0.8-mW 55$\mathrm{GHz}$ dual-injection-locked CMOS frequency 
divider. IEEE Trans. Microw. Theory Tech. 56(3), 620-625 (2008)

[37] J. van der Tang, High-Frequency Oscillator Design for Integrated Transceivers, Ph.D. dissertation, Eindhoven University of Technology, 2002

[38] A. Hajimiri, S. Limotyrakis, T.H. Lee, Jitter and phase noise in ring oscillators. IEEE J. Solid-State Circuits 34(6), 790-804 (1999)

[39] J.O. Plouchart, K. Jonghae, N. Zamdmer, M. Sherony, T. Yue, Y. Meeyoung, M. Talbi, A. Ray, L. Wagner, A $31 \mathrm{GHz}$ CML ring VCO with 5.4 ps delay in a $0.12 \mathrm{~mm}$ SOI CMOS technology. IEEE European Solid-State Circuits Conference, Sept. 2003, pp. 357-360

[40] W. Hui, A. Hajimiri, Silicon-based distributed voltage-controlled oscillators. IEEE J. Solid- State Circuits 36(3), 493-502 (2001)

[41] L. Lianming, P. Reynaert, M. Steyaert, A 90nm CMOS mm-wave VCO using an LC tank with inductive division. IEEE European Solid-State Circuits Conference, Sept. 2008, pp. 238-241

[42] K.W. Tang, S. Leung, N. Tieu, P. Schvan, S.P. Voinigescu, Frequency scaling and topology comparison of millimeter-wave CMOS VCOs. IEEE Compound Seminconductor Integrated Circuit Symposium, Nov. 2006, pp. $55-58$

[43] B. Heydari, M. Bohsali, E. Adabi, A.M. Niknejad, Millimeter-wave devices and circuit blocks up to $104 \mathrm{GHz}$ in $90 \mathrm{~nm}$ CMOS. IEEE J. Solid-State Circuits 42(12), 2893-2903 (2007)

[44] L.M. Franca-Neto, R.E. Bishop, B.A. Bloechel, $64 \mathrm{GHz}$ and $100 \mathrm{GHz}$ VCOs in 90 nm CMOS using optimum pumping method. IEEE International Solid-State Circuits Conference, Feb. 2004, pp. 444-538

[45] C. Changhua, K.O. Kenneth, Millimeterwave voltage-controlled oscillators in 0.13mm CMOS technology. IEEE J. Solid-State Circuits 41(6), 1297-1304 (2006)

[46] A.P. van der Wel, S.L.J. Gierkink, R.C. Frye, V. Boccuzzi, B. Nauta, A robust $43-\mathrm{GHz}$ VCO in CMOS for OC-768 SONET applications. IEEE J. Solid-State Circuits 39(7), 1159-1163 (2004)
[47] D.B. Leeson, A simple model of feedback oscillator noise spectrum. Proc. IEEE 54(2), $329-330$ (1966)

[48] J.J. Rael, A.A. Abidi, Physical processes of phase noise in differential LC oscillators. IEEE Custom Integrated Circuits Symposium, May 2000, pp. 569-572

[49] A. Hajimiri, T.H. Lee, A general theory of phase noise in electrical oscillators. IEEE J. Solid- State Circuits 33(2), 179-194 (1998)

[50] J. Craninckx, M. Steyaert, Low-noise voltagecontrolled oscillators using enhanced LC tanks. IEEE Trans. Circuits Syst. II: Analog Digit. Signal

[51] B. Razavi, A study of phase noise in CMOS oscillators. IEEE J. Solid-State Circuits 31(3), 331- 343 (1996)

[52] E. Hegazi, H. Sjoland, A.A. Abidi, A filtering technique to lower LC oscillator phase noise. IEEE J. Solid-State Circuits 36(12), 1921-1930 (2001)

[53] R. Aparicio, A. Hajimiri, A CMOS differential noise-shifting Colpitts VCO. IEEE International Solid-State Circuits Conference, Feb. 2002, pp. 288-

[54] M Vineeth Bhat, Siddanth Jain, M. P. Srivatsa, M. Nithin, Harish M. Kittur. "Design of low phase noise voltage controlled oscillator for phase locked loop" , 2017 International conference on Microelectronic Devices, Circuits and Systems (ICMDCS), 2017

[55] L.-F. Tanguay, M. Sawan, Y. Savaria. "A veryhigh output impedance charge pump for low voltage low-power PLLs" , Microelectronics Journal, 2009

[56] Zhao, Bo, and Huazhong Yang. "SupplyNoise Interactions Among Submodules Inside a Charge- Pump PLL", IEEE Transactions on Very Large Scale Integration (VLSI) Systems, 2015.

[57] Hammad M Cheema, Reza Mahmoudi, Arthur H.M. van Roermund, "60 GHz CMOS Phase Locked Loops", Springer 2010.

[58] W. Hu, L. Chunglen, X. Wang, Fast frequency acquisition phase-frequency detector with zero blind zone in PLL. IET Electron. Lett. 43(19), 1018-1020 (2007) 
[59] M. Mansuri, D. Liu, C.K.K. Yang, Fast frequency acquisition phase-frequency detectors for Gsamples/s phase-locked loops. IEEE J. Solid-State Circuits 37(10), 1331-1334 (2002)

[60] G.B. Lee, P.K. Chan, L. Siek, A CMOS phase frequency detector for charge pump phase locked loop. IEEE Midwest Symposium on Circuits and Systems, 1999, pp. 601-604

[61] H.O. Johansson, A simple precharged CMOS phase frequency detector. IEEE J. SolidState Circuits 33(2), 295-299 (1998)

[62] H. Kondoh, H. Notani, T. Yoshimura, H. Shibata, Y. Matsuda, A 1.5-V 250-MHz to 3.0$\mathrm{V} 622-\mathrm{MHz}$ operation CMOS phase-locked loop with precharge type phase-frequency detector. IEICE Trans Electron E78-C(4), 381-388 (1995)

[63] Nithin M, Harish M Kittur, Design of a beyond millimeter wave oscillator in $22 \mathrm{~nm}$ Bulk CMOS technology. International Journal of Engineering and Advanced Technology (IJEAT) Volume-9 Issue-1S3, December 2019 . 